\title{
Three-bottle exponential decay infusion: A novel intravenous infusion strategy for drugs which conform to three-compartment model with observations on propofol kinetics
}

\author{
Xiao Hui Huang1, Hai Tang Xie'², Kun Wang3, Ji Han Huang3 and Rui Yuan Sun² \\ ${ }^{1}$ Center of Pharmacokinetics, College of Pharmacy, Anhui Medical University, Hefei 230 032, Anhui, China; ${ }^{2}$ Anhui \\ Provincial Center for Drug Clinical Evaluation, Institute of Clinical Pharmacology and Pharmacy, Yijishan Hospital of \\ Wannan Medical College, Wuhu 241 001, Anhui, China; ${ }^{3}$ Department of pharmacometrics, Center for Drug Clinical \\ Research, Shanghai University of Chinese Medicine, Shanghai 20123 , China.
}

\begin{tabular}{|c|c|}
\hline \multicolumn{2}{|l|}{ Article Info } \\
\hline Received: & 21 September 2012 \\
\hline Accepted: & 22 September 2012 \\
\hline Available Online: & 12 October 2012 \\
\hline \multicolumn{2}{|c|}{ DOI: 10.3329/bjp.v7i4.11908 } \\
\hline \multicolumn{2}{|c|}{$\begin{array}{l}\text { Cite this article: } \\
\text { Huang XH, Xie HT, Huang JH, Sun } \\
\text { RY. Three-bottle exponential decay } \\
\text { infusion: A novel intravenous infusion } \\
\text { strategy for drugs which conform to } \\
\text { three-compartment model with obser- } \\
\text { vations on propofol kinetics. Bangla- } \\
\text { desh J Pharmacol. 2012; } 7: 223-35 \text {. }\end{array}$} \\
\hline
\end{tabular}

\section{Abstract}

The aim of this study is to develop a novel three-bottle exponential decay infusion method to achieve the steady state concentration rapidly for drugs which conform to three-compartment model. The mathematical model and dosing regimen of three-bottle exponential decay infusion were developed. The input concentration of this method is a multi-exponential decay variable. With this method, blood concentration increased very rapidly to plateau value in the early phase of infusion and the target plateau concentration can be achieved much more quicker (approximately 10 to 35 times) than conventional constant rate infusion. This procedure was verified in rabbits and dogs and compared with conventional constant rate infusion with observations on propofol kinetics. This new quantitative methodology may provide a novel intravenous infusion strategy for drugs which conform to three-compartment model and can be used for the program design of infusion pump.

\section{Introduction}

Constant rate infusion (CRI) is very commonly used in clinical practice. For drugs with a long elimination halflife, the time at which the steady state is achieved might be very long. To overcome this drawback, a loading dose is frequently used to achieve the steady state as early as possible. It is easy to calculate the loading dose and infusion rate for one-compartment model drugs, but such calculations are rather complicated for two- or three-compartment model drugs (Gibaldi et al., 1982). Some scholars have proposed different dosing regimens to solve such problem for two-compartment drugs (Kruger-thiemer et al., 1968; Wagner et al., 1974; Boyes et al., 1971; Mitento et al., 1972; Zhu et al., 1980; Sun et al., 1983; Sun et al., 2004; Xie et al., 2011). Each method has its own advantages and disadvantages (Xie et al., 2011). However, the pharmacokinetic features of some drugs, such as propofol (Puri et al., 2012; Ye et al., 2012), raltitrexed (Blair et al., 2004; Clarke et al., 2000), thiopental (Russo et al., 1998), midazolam (Hardmeier et al., 2012), and digoxin (Krishna et al., 2011) etc. should be effectively described with the use of the three -compartment open-system model. For such drugs, the target plateau concentrations often can not be achieved rapidly enough by conventional CRI method, which may delays the therapeutic effects (van den Nieuwenhuyzen et al., 2000; Xie et al., 2011). According to the literature search using MEDLINE, there is hardly satisfactory quantitative methodology to address this problem. 


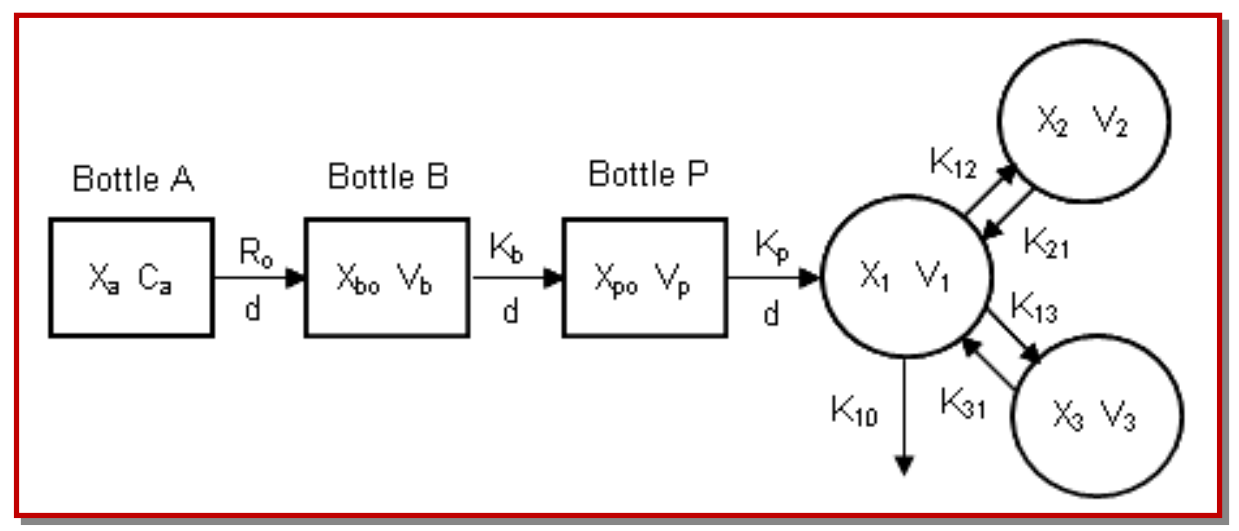

Figure 1: Diagram of three-bottle exponential decay infusion. $\mathrm{R}_{\mathrm{o}}, \mathrm{k}_{\mathrm{b}}$ and $\mathrm{k}_{\mathrm{p}}$ are the infusion rate constants of bottles $\mathrm{A}, \mathrm{B}$ and $\mathrm{P}$, respectively; $d$ is the infusion rate; $X_{a}, X_{b 0}$ and $X_{p 0}$ are the drug amounts of bottles $A, B$ and $P$, respectively; $V_{b}$ and $V_{p}$ are the drug volumes of bottles $B$ and $P$, respectively. $X_{1}, X_{2}$ and $X_{3}$ are the drug amounts of central compartment and two peripheral compartments, respectively. $\mathrm{K}_{12}$ and $\mathrm{K}_{13}$ are distribution rate constants from central compartment to the peripheral compartments. $\mathrm{K}_{21}$ and $\mathrm{K}_{31}$ are distribution rate constants from the peripheral compartments to the central compartment; $K_{10}$ is elimination rate constant for loss of drug from the central compartment

In our previous study, we successfully developed a two -bottle exponential decay infusion method for drugs which conform to two-compartment model and verified it in dogs and healthy volunteers (Song et al., 1983). In the present study, a quantitative methodology on the basis of the three-compartment open-system model is described by which steady state concentration can be achieved much more rapidly than conventional CRI method by exponentially changing the infusion concentration during the infusion process. This quantitative method may provide a novel intravenous infusion strategy for drugs which conform three-compartment model and can be used for the program design of infusion pump.

\section{Materials and Methods}

\section{Mathematical model and dosing regimen}

Figures 1 show the mathematical model of the threebottle exponential decay infusion. Related equations of dosing regimen and the concentration-time courses are described in Table I. Mathematical proof of these equations was attached in appendix. For the purposes of this discussion, it is postulated that the body behaves as a three-compartment open-system model with components shown in Figure 1. The model where elimination occurs from a central compartment which is reversibly connected to two peripheral compartments. The rates of transferring between compartments and the rate of drug elimination from the central compartment are considered to follow first-order kinetics.

When carrying out this method, three infusion bottles $(\mathrm{A}, \mathrm{B}$ and $\mathrm{P})$ are connected in series. The drug concen- tration $\left(C_{a}\right)$ of bottle $A$ were set equal to that of the conventional CRI method. The intial concentration of bottle $B\left(\mathrm{C}_{\mathrm{b} 0}\right)$ is set higher than $\mathrm{Ca}$ and the intial concentration of bottle $\mathrm{P}\left(\mathrm{C}_{\mathrm{p} 0}\right)$ is set higher than $\mathrm{C}_{\mathrm{b} 0}$. The concentrations, and drug volumes and amounts of these three infusion bottles can easily calculated according to the equations in Table I. And this dosing regimen can be easily written in a computer program. From equation 1, we can see that if the infusion rates of these three bottle remain constant, the input concentration of TBEDI is a multi-exponential decay variable. Initially, the concentration in bottle $\mathrm{P}$ (the highest concentration), was directly infused to the central compartment. The concentrations in bottle $\mathrm{B}$ and $\mathrm{P}$ are diluted in an exponential way all the time. As the infusion time tends to infinite or lasts long enough, the concentrations in the three bottles can be approximately equal to that of $C_{a}$ (the lowest concentration). At the very beginning of TBEDI, the blood concentration can increased very rapidly to plateau concentration and maintained at this level as long as desired.

\section{Experimental procedure}

Rabbits ( + and $\hat{\sigma}, \mathrm{n}=6$, weighing $2.0 \pm 0.2 \mathrm{~kg}$ ) and dogs ( + and $\hat{\jmath}, \mathrm{n}=3$, weighing $5.0 \pm 0.2 \mathrm{~kg}$ ) were obtained from the Animal Breeding Center of Wannan Medical College (Anhui Province, China). The study protocol was approved by the Ethical Committee for Animal Experimentation of Wannan Medical College. Propofol emulsion for intravenous injection $(10 \mathrm{mg} / 1 \mathrm{~mL}, 200$ mg per vial) was purchased from Zeneca company. 5\% Glucose was obtained from the Pharmaceutical Department of Yijishan Hosptical Affiliated to Wannan Medical College. 
Table I

Equations of dosing regimen for three-bottle exponential decay infusion

The time course of plasma concentration in bottle P :

$C_{p}(t)=C_{a}+\left(C_{b o}-C_{a}\right) \frac{V_{b}}{V_{b}-V_{p}} e^{-\frac{d}{V b} t}+\left[\left(C_{p o}-C_{a}\right)-\left(C_{b o}-C_{a}\right) \frac{V_{b}}{V_{b}-V_{p}}\right] e^{-\frac{d}{V p} t}$

(1)

When $\mathrm{t}=0, \mathrm{C} \mathrm{p}(\mathrm{t})=\mathrm{C}_{\mathrm{po}}$; when $t \rightarrow \infty, \mathrm{C}_{\mathrm{p}}(\mathrm{t})=\mathrm{C}_{\mathrm{a}}$

The time course of plasma concentration in central compartment :

$C_{1}(t)=\frac{d C a}{K_{10} V_{1}}\left(1-e^{-\pi t}\right)$

When $t \rightarrow \infty$,

$$
C_{1}(\infty)=\frac{C_{a} d}{K_{10} V_{1}}=C_{\mathrm{ss}}
$$

Concentration in bottle A:

$$
C_{a}=\frac{C_{s s} V_{1} K_{10}}{d}
$$

Initial drug concentration in bottle B:

$$
C_{b o}=\frac{\operatorname{CadK}_{31}}{\alpha \beta}\left(\alpha+\beta-K_{31}\right)
$$

$$
V_{b}=\frac{d}{K_{31}}
$$

Drug volume of bottle B:

Initial drug amount in bottle $\mathrm{B}: \quad \mathrm{X}_{\mathrm{b} 0}=\mathrm{C}_{\mathrm{b} 0} \times \mathrm{V}_{\mathrm{b}}$

$$
C_{p o}=\frac{C_{a} \pi}{K_{10}}
$$

Initial drug concentration in bottle P:

Drug volume of bottle P:

$$
V_{p}=\frac{d}{K_{21}}
$$

Rabbits and dogs were administered by three kinds of drug administration methods (i.e., intravenous injecttion, TBEDI and CRI) with a one-week wash-out period for each method. The experimental procedures were as follows: First, propofol in a dose of $10 \mathrm{mg} / \mathrm{kg}$ was injected in rabbits and dogs to study the kinetic behavior of this drug and obtain the individual pharmacokinetic parameters. The information obtained was used to calculate the dosing regimen of TBEDI and CRI. Blood samples were collected before and at 1, 2, $3,4,5,6,8,10,15,20,25,30,35,40,45,50,60 \mathrm{~min}$ following the bolus intravenous injection of propofol. Then two infusion methods, TBEDI and CRI, were carried out respectively and compared. When using TBEDI method, The different concentrations and drug volumes for the bottle $\mathrm{A}, \mathrm{B}$ and $\mathrm{P}$ were calculated according to the equations in Table I based on individual pharmacokinetic parameters. When using CRI method, only one bottle (bottle A) was used. Blood samples were drawn according to the exact time schedules $(0,2,4,6,8,10,12,15,18,20,25,30 \mathrm{~min}$ for TBEDI, and $0,2,6,10,15,20,25,30 \mathrm{~min}$ for CRI.). When carrying out and comparing these two infusion methods, the desired steady state plasma drug concentration was set at $3 \mu \mathrm{g} / \mathrm{mL}$. The infusion device was connected to a constant flow pump and the flow rate was adjusted at $1 \mathrm{~mL} / \mathrm{min}$. During the above procedures, the ampule of propofol was shaked for at least $1 \mathrm{~min}$ before use and the appropriate dose was aspirated accurately according to the body weight of the rabbit or dog. The rabbits or dogs were fixed properly on the operating table and all the animals had venous canula inserted for sampling. Venous blood propofol concentrations were determined by HPLC. DAS 3.0 program was used to analyze the pharmacokinetic data. DAS is the most widely used 

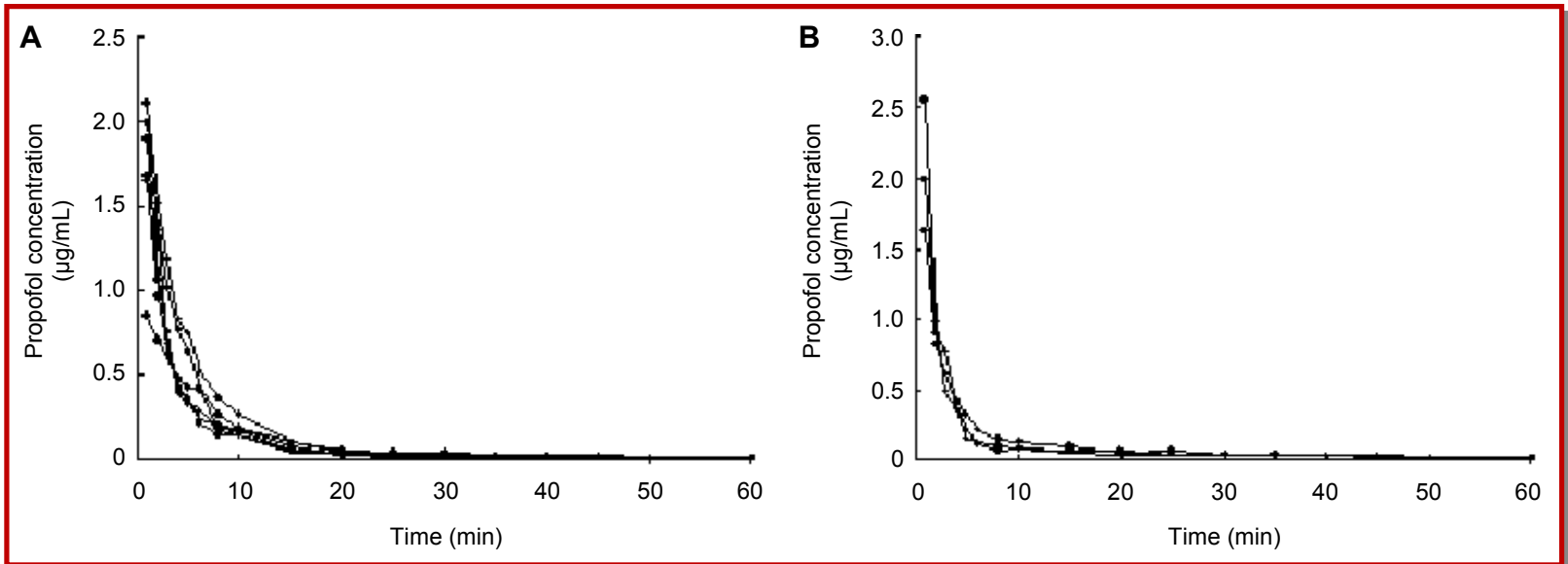

Figure 2: Measured plasma propofol concentrations in dogs following the bolus intravenous injection at $10 \mathrm{mg} / \mathrm{kg}$. (A: six rabbits; B: three dogs)

pharmacokinetic software in mainland China.

Blood samples were collected in heparinized centrifugation tubes and stored at $4^{\circ} \mathrm{C}$ until assay. After adding $0.1 \mathrm{~mL}$ blood sample and $0.1 \mathrm{~mL}$ acetonitrile in $1 \mathrm{~mL}$ plastic centrifugation tube, the mixture then was blended on an inversion mixer for $10 \mathrm{sec}$ and centrifugated at $15,000 \mathrm{~g}$ for $5 \mathrm{~min}$. A $100 \mathrm{uL}$ volume of the supernatant was taken for HPLC analysis. HPLC system (Waters Assoc.) consisted of a Waters 515 MS system controller, a Waters 717 plus automatic sample injector and a 474 fluorimetric detector. Chromatographic conditions: The HPLC mobile phase consisted of acetonitrile and distilled water $(65: 35, \mathrm{v} / \mathrm{v}) . \mathrm{pH}$ was adjusted to 4.0 using acetic acid. Chromatographic separation was carried out by a $200 \times 4.6 \mathrm{~mm}$ column packed with silane particle (C18) which was used at ambient temperature of $30^{\circ} \mathrm{C}$. The excitation and emission wavelenths were set at 216 and $370 \mathrm{~nm}$ respectively. The linearity of the curve in the range of 5 to $5,000 \mathrm{ng} / \mathrm{mL}$ and the relative standard deviations in this concentration range were less than $10 \%$. The limit of quantification proved to be $0.4 \mathrm{mg} / \mathrm{L}$. Plasma drug concentrations were calculated according to the peak area of the samples.

\section{Results}

The plasma concentration-time profiles of propofol following the bolus intravenous injection are shown in Figure 2. The pharmacokinetics of propofol were best fitted to a three-compartment open model. The main parameters are summarized in Table II.

Table III shows the dosing regimen of the present study. The different concentrations and drug volumes for the bottle A, B and P were calculated and dispensed according to the equations in Table I and individual pharmacokinetic parameters in Table II. The results are presented graphically for each animal. Figure 3 and 4 show the predicted and measured propofol concentration-time profiles of TBEDI and conventional CRI methods in rabbits and dogs. In general, the observed plasma values agree well with the predicted concentrations. The results demonstrate that the time of obtain 90 per cent plateau concentration is approximately 4 to 6 min by TBEDI for all the subjects. On the other hand, for conventional mono-bottle infusion, it would require up to $30 \mathrm{~min}$ to reach 50 per cent and estimated approximately 59 to $143 \mathrm{~min}$ to reach 90 per cent of plateau concentration. The target concentration was maintained for $30 \mathrm{~min}$ in our study and could be as long as desired. The result also demonstrates that the optimal anesthetic target, or adequate depth and satisfactory quality of anesthesia were achieved with maintain concentration of between 2.5 and $4.7 \mu \mathrm{g} / \mathrm{mL}$ of propofol in rabbits and dogs. With this method, the speed of achieving the 90 per cent plateau concentration was more than 10 to 35 times faster than that of the conventional constant rate infusion in the present study.

\section{Discussion}

Many therapeutic agents can be applied more predictably and effectively if a constant plasma concentration is maintained. Many clinical situations often demand that the steady-state concentration be reached as rapidly as possible. It is often desirable in such cases to administer an intravenous loading dose just before starting the intravenous infusion. For two-compartment drugs, the calculation of loading dose and fusion rate is 
Table II

Main pharmacokinetic parameters of propofol in rabbits and dogs following bolus injection at $10 \mathrm{mg} / \mathrm{kg}$

\begin{tabular}{|lcccccccccc|}
\hline Parameters & $\Pi$ & $\mathrm{a}$ & $\beta$ & $\begin{array}{c}\mathrm{V}_{\mathrm{c}} \\
(\mathrm{L} / \mathrm{mg})\end{array}$ & $\begin{array}{c}\mathrm{k}_{12} \\
\left(\mathrm{~min}^{-1}\right)\end{array}$ & $\begin{array}{c}\mathrm{k}_{21} \\
\left(\mathrm{~min}^{-1}\right)\end{array}$ & $\begin{array}{c}\mathrm{k}_{13} \\
\left(\mathrm{~min}^{-1}\right)\end{array}$ & $\begin{array}{c}\mathrm{k}_{31} \\
\left(\mathrm{~min}^{-1}\right)\end{array}$ & $\begin{array}{c}\mathrm{k}_{10} \\
\left(\mathrm{~min}^{-1}\right)\end{array}$ \\
\hline Rabbits & 1.822 & 0.202 & 0.016 & 2.439 & 0.810 & 0.643 & 0.012 & 0.016 & 0.559 \\
R1 & 0.827 & 0.368 & 0.039 & 3.406 & 0.093 & 0.584 & 0.139 & 0.056 & 0.363 \\
R2 & 0.458 & 0.157 & 0.033 & 3.382 & 0.079 & 0.263 & 0.018 & 0.036 & 0.251 \\
R3 & 0.613 & 0.169 & 0.021 & 2.224 & 0.624 & 0.561 & 0.024 & 0.014 & 0.597 \\
R4 & 0.595 & 0.404 & 0.042 & 3.695 & 0.101 & 0.714 & 0.100 & 0.417 & 0.323 \\
R5 & 1.213 & 0.202 & 0.040 & 3.031 & 0.089 & 0.303 & 0.024 & 0.041 & 0.347 \\
R6 & 0.921 & 0.250 & 0.032 & 3.030 & 0.299 & 0.511 & 0.053 & 0.097 & 0.407 \\
Mean & 0.469 & 0.098 & 0.010 & 0.533 & 0.300 & 0.169 & 0.049 & 0.144 & 0.127 \\
SD & & & & & & & & & \\
Dogs & 0.640 & 0.229 & 0.021 & 3.460 & 0.050 & 0.263 & 0.176 & 0.032 & 0.370 \\
D1 & 1.460 & 0.555 & 0.033 & 1.211 & 0.182 & 0.736 & 0.302 & 0.046 & 0.782 \\
D2 & 1.202 & 0.347 & 0.032 & 2.222 & 0.099 & 0.514 & 0.229 & 0.040 & 0.502 \\
D3 & 1.101 & 0.377 & 0.029 & 2.298 & 0.110 & 0.504 & 0.236 & 0.039 & 0.551 \\
Mean & 0.419 & 0.165 & 0.007 & 1.126 & 0.067 & 0.237 & 0.063 & 0.007 & 0.210 \\
SD & & & & & & & & & \\
aR1 to R6 and D1 to D3 denote six rabbits and three dogs, respectively & & & & & &
\end{tabular}

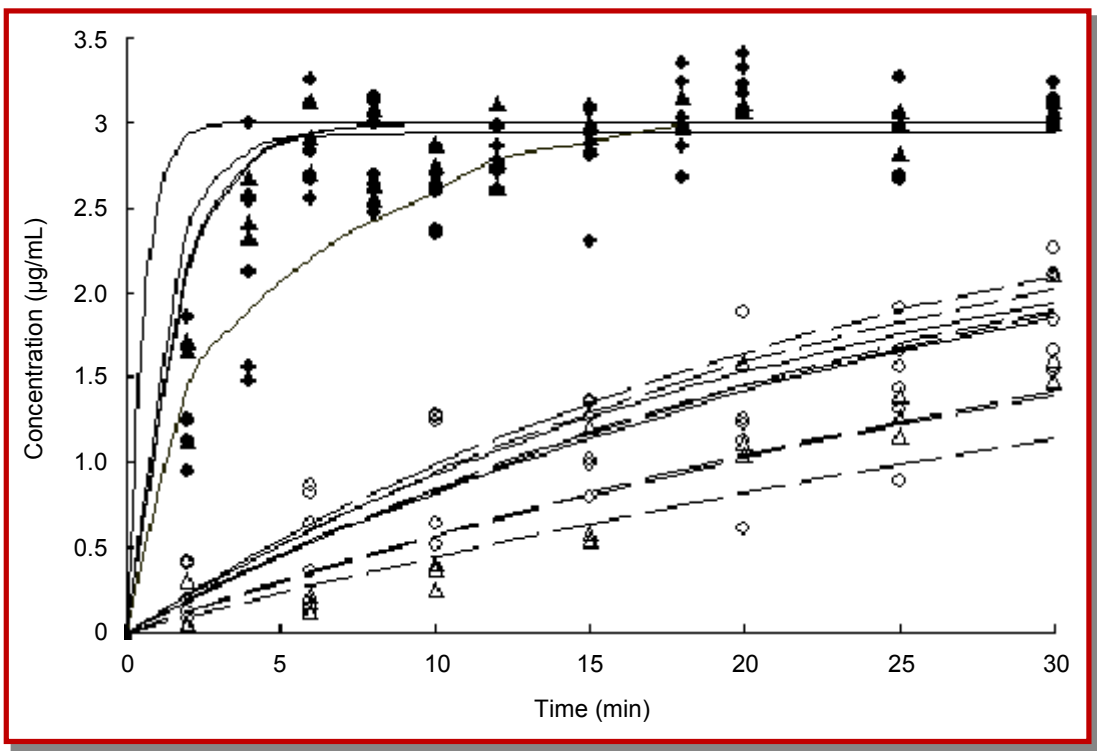

Figure 3: The comparision of predicted and measured propofol concentration-time profiles of TBEDI and CRI methods in rabbits and dogs. Smoothed and broken lines denote the predicted concentrations of TBEDI and CRI methods, respectively. Filled and hollow circles denote the measured concentrations of TBEDI and CRI in rabbits, respectively. Filled and hollow triangles denote the measured concentrations of TBEDI and CRI in dogs, respectively

relatively complicated. Some theoretical and experimental studies have been published attempting to provide possible solutions. Each method has its own advantages and disadvantages. For example, Boyes's method is relatively safe, but this approach has the drawback of a minimum concentration which may be well below that required, followed by a slow return to plateau values (Boyes et al., 1971; Sun et al., 2004). With the method developed by Mitenko (Mitenko et al., 1972), the concentration during the early infusion phase is very high, sometimes even the 1.5 to 5 times to target concentration, which may often exceed the safe limits of 
Table III

Dosing regimen of TBEDI in the present study

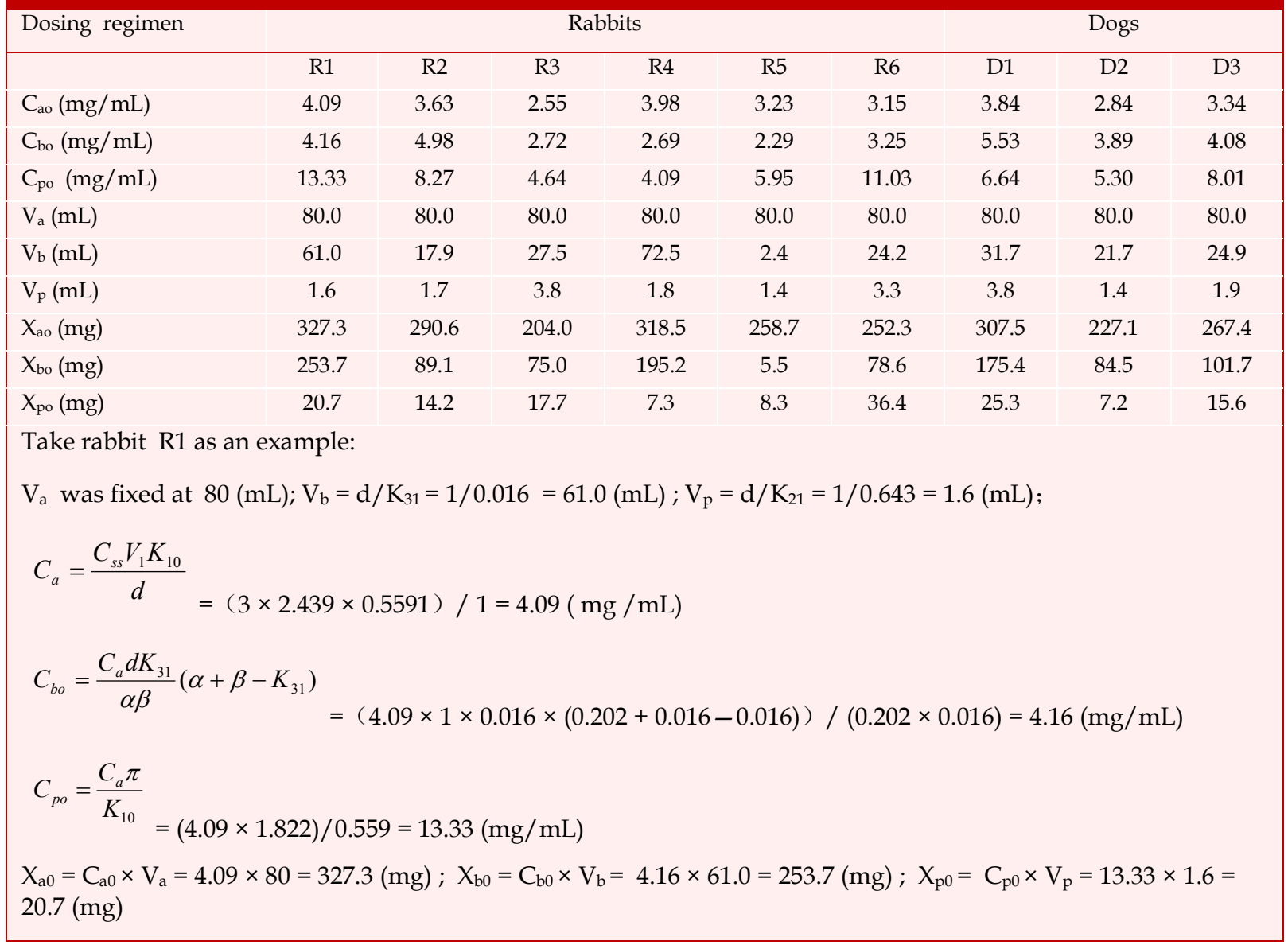

drugs with small therapeutic indexes. Some researches proposed regimens similar to these two methods, but still could not solve the problem of avoiding the high concentrations during early infusion phase (Wagner et al., 1974; Chau, 1977; Zhu, 1980). In our previous study (Song et al., 1983), we successfully developed a doublebottle exponential decay infusion method with observations on gentamicin kinetics. With this method, the rate of infusion is exponential with time and steady-state concentration can be obtained very rapidly for one- or two-compartment models. Actually, two-bottle exponential decay infusion can be seen as the particular case of the TBEDI method described in this study. This procedure was verified in 12 dogs and 8 normal male volunteers and was more safe compared with Boyes', Mitenko' and Zhu' methods.

However, there are a number of effective but highly toxic drugs that do manifested multi-exponential characters which are too simplistic to explain using one- or two-compartment model. In particular, it has been pointed out that the pharmacokinetic characteristics of many anesthetic drugs can be effectively described with the use of three-compartment open-system model (Nieuwenhuyzen et al., 2000). In such cases, there is considerable potential for inadequate or excessively high drug concentration in the blood in clinical practice. On the other hand, with conventional CRI infusion method, target plateau value can be achieved slowly only after 4-5 elimination half-lives have passed which is clearly unsuitable for anaesthetic drugs in many clinical situations. According to the literature search using MEDLINE, there is hardly satisfactory quantitative methodology to address this problem.

A possible solution is offered in this paper. This method can be useful for drugs which conform to threecompartment open model, especially those with long half-lives and small therapeutic index. Only a programmable hand calculator is needed to calculate the 

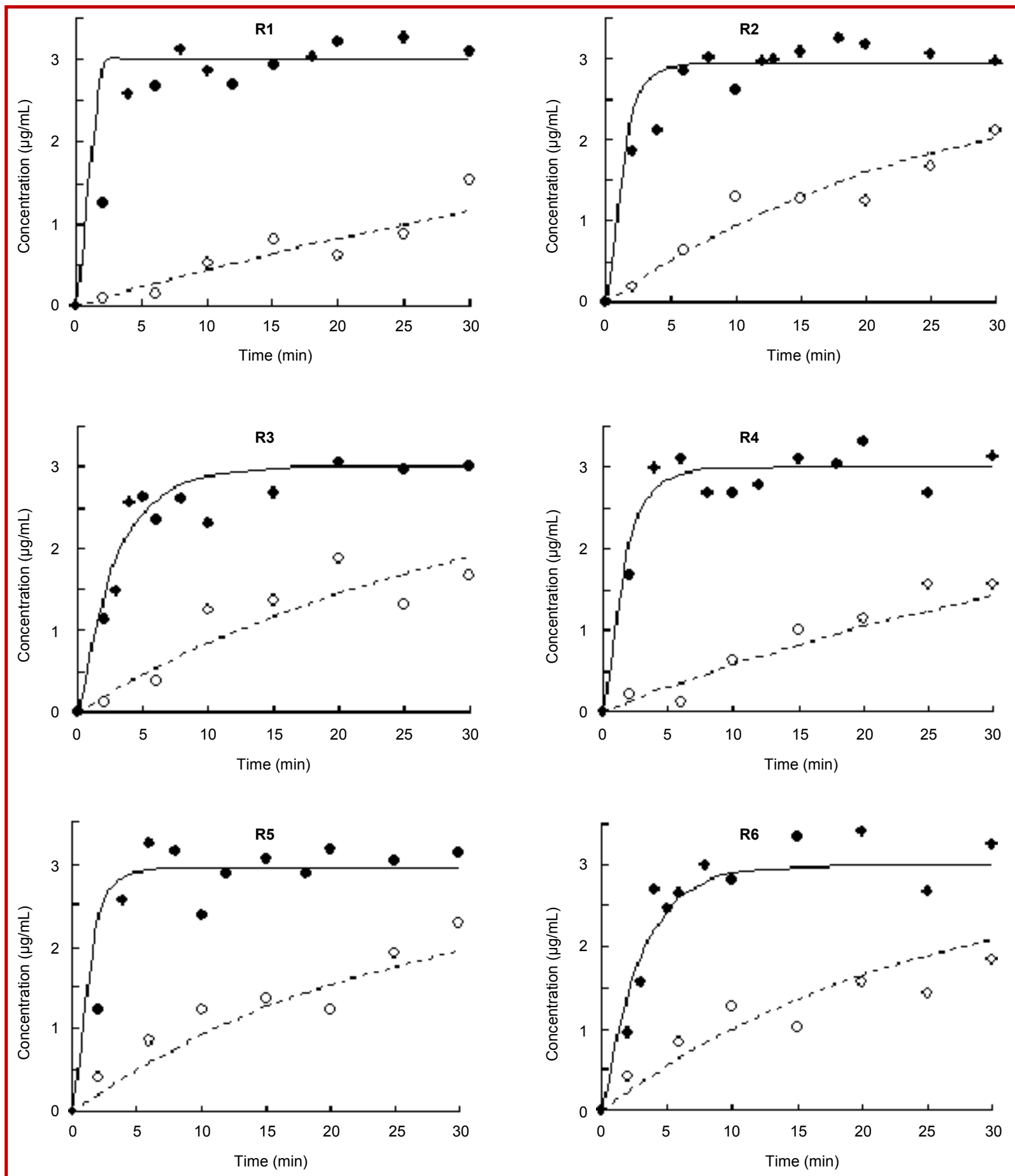

Figure 4: The comparison of predicted and measured propofol concentration-time profiles of TBEDI and MBI methods in each experimental animal. R1 to R6 and D1 to D3 denote six rabbits and three dogs, respectively. The symbols are defined in Figure 3

dosing regimen. With this method, a specified steadystate plasma concentration can be rapidly obtained by combining three bottles in series with exponential decay of drug concentration. The time needed to achieve the steady state can be more than 10 to 35 times faster than that of the conventional constant rate infusion. This method is especially suitable for anesthetic agents, such as propofol and alfentanil. Optimal therapy with such drugs requires appropriate dosing regimen to safely obtain the desired clinical effects 


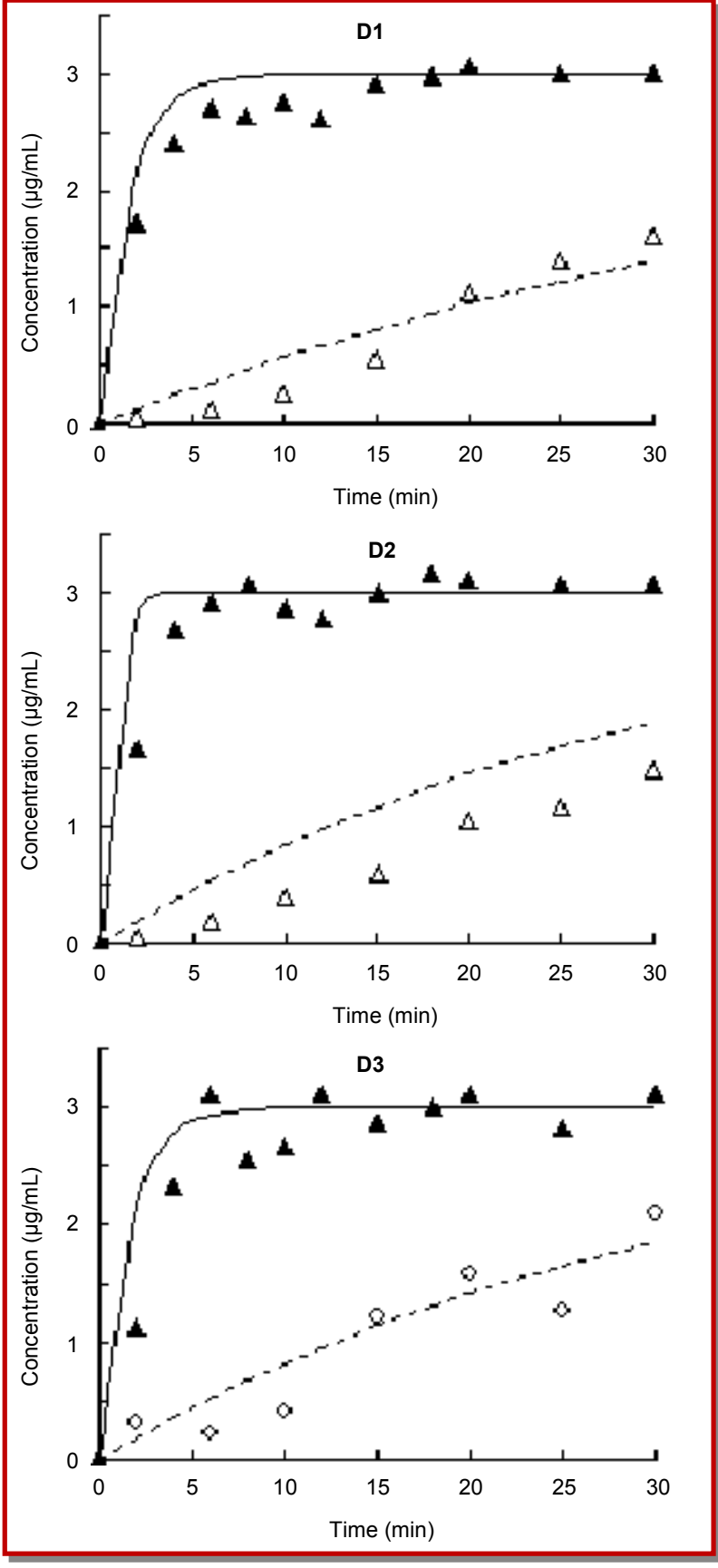

Figure 4: The comparison of predicted and measured propofol concentration-time profiles of TBEDI and MBI methods in each experimental animal. R1 to R6 and D1 to D3 denote six rabbits and three dogs, respectively. The symbols are defined in Figure 3 (Cont.)

without causing toxicity.

In clinical application, to ensure the liquid medicines in the bottles $\mathrm{B}$ and $\mathrm{P}$ be blend completely, a electromagnetic stirring device could be used. And constant rate infusion pump could be used to control the infu- sion rate to be remained constant and ensure accurate delivery. However, according to our experi-ences in previous study (Song et al., 1983), even if only the clamps are used to control the infusion rate in clinical practice, the satisfactory result could also be obtained. Actually, even if the liquid medicines in bottles B and P can not be diluted completely, the concentrations during the early infusion phase of this method are still much lower than those of Mitento' and Zhu' method (Song and Sun, 1983; Xie et al., 2011). It should be also noted that, when applying this method, in the early phase of infusion the drug amount into the body is larger than that of CRI, and drug concentrations rise relatively quickly. So, for intense irritant drugs and those with large toxic effects on cardiovascular or central nervous system, this method should be used with caution. In addition, The three-bottle exponential decay infusion scheme may also be applied to maintain the concentration of drug in a particular compartment of a multi-compartment model (e.g., the compartment representing the site of drug effect), but the mathematic models may be more complicated.

\section{Conclusion}

The three-bottle exponential decay infusion quantitative method, with which drug concentration plateaus can be achieved much more rapidly compared with conventional constant rate infusion, may provide a novel intravenous infusion strategy for drugs which conform to three-compartment model and can be used for the program design of infusion pump.

\section{Contribution}

Xiao Hui Huang and Hai Tang Xie contributed equally to this work

\section{References}

Blair EY, Rivory LP, Clarke SJ. Population pharmacokinetics of raltitrexed in patients with advanced solid tumours. Br J Clin Pharmacol. 2004; 57: 416-26.

Boyes RN, Scott DB, Jebson PJ, Godman MJ, Julian DG. Pharmacokinetics of lidocaine in man. Clin Pharmacol Ther. 1971; 12: 105-16.

Chau NP. linear pharmacokinetics: Calculation of the loading dose for two-compartment models with intermittent infusions. J Pharmacokinet Biopharm. 1977; 5: 391-403.

Clarke SJ, Beale PJ, Rivory LP. Clinical and preclini- 
cal pharmacokinetics of raltitrexed. Clin Pharmacokinet. 2000; 39: 429-43.

Gibaldi M, Perrier D. Pharmacokinetics. 2nd ed. Marcel Dekker, New York, 1982.

Hardmeier M, Zimmermann R, Rüegg S. Intranasal midazolam: Pharmacokinetics and pharmacodynamics assessed by quantitative EEG in healthy volunteers. Clin Pharmacol Ther. 2012; 91: 856-62.

Krishna R, Stypinski D, Ali M. Lack of an effect of anacetrapib on the pharmacokinetics of digoxin in healthy subjects. Biopharm. Drug Dispos. 2011; 32: 525-29.

Krüger-Thiemer E. Continuous intravenous infusion and multi -compartment accumulation. Eur J Pharmacol. 1968; 4: 31724.

Mitenko PA, Ogilvie RI. Rapidly achieved plasma concentration plataus with observations on theophylline kinetics. Clin Pharmacol Ther. 1972; 13: 329-25.

Puri A, Medhi B, Panda NB. Propofol pharmacokinetics in young healthy Indian subjects. Indian J Pharmacol. 2012; 44: 402-06.

Russo H, Bressolle F. Pharmacodynamics and pharmaco- kinetics of thiopental. Clin Pharmacokinet. 1998; 35: 95-134.

Song JG, Sun RY. Pharmacokinetics of double-bottle intravenous infusion. Acta Pharmaceutica Sinica. 1983; 183: 732-40.

Sun RY, Zheng QS. New concepts of mathematical pharmacology. People's Medical Publishing House, 2004.

van den Nieuwenhuyzen MC, Engbers FH, Vuyk J, Burm AG. Target-controlled infusion systems: Role in anaesthesia and analgesia. Clin Pharmacokinet. 2000; 38: 181-90.

Wagner JG. A safe method for rapidly achieving plasma concentration plateaus. Clin Pharmacol Ther. 1974; 16: 691700 .

Xiao S. Guiding clinical anesthesia prescription by the principle of pharmacokinetics. Mod Hosp. 2008; 8: 7-9.

Xie HT, Huang XH, Shi J. Quantitative pharmacology and drug development. People's Military Medical Press, 2011.

Ye HB, Li JH, Rui JZ, Zheng H, Zhang XA, Chi XJ, Chen WY, $\mathrm{Xu}$ JG. Propofol pharmacokinetics in China: A multicentric study. Indian J Pharmacol. 2012; 44: 393-97.

Zhu JB. Mathematical analysis concerning the dosage regimen of intravenous infusion of two-compartment model drugs. Acta Pharmaceutica Sinica. 1980; 15: 741-49. 


\section{Appendix: Proof of Equations (1)-(10) in Table I}

The following differential equations may be written for the change in amount of drug in the bottle B and P with time:

$$
\begin{aligned}
& \frac{d X_{b}}{d t}=R_{o}-K_{b} X_{b} \\
& \frac{d X_{p}}{d t}=K_{b} X_{b}-K_{p} X_{p}
\end{aligned}
$$

The solution of the differential equations is

$$
X_{p}(t)=X_{p o}\left\{\frac{R_{o} K_{b}}{X_{p o} K_{b} K_{p}}+\frac{K_{b}{ }^{2}-K_{b}{ }^{2}\left(1+\frac{X_{b o}}{X_{p o}}\right)+\frac{R_{o} K_{b}}{X_{p o}}}{K_{b}\left(K_{b}-K_{p}\right)} e^{-K b t}+\frac{K_{p}{ }^{2}-K_{p} K_{b}\left(1+\frac{X_{b o}}{X_{p o}}\right)+\frac{R_{o} K_{b}}{X_{p o}}}{K_{p}\left(K_{p}-K_{b}\right)} e^{-K p t}\right\}
$$

which can be written in concentration terms:

$$
C_{p}(\mathrm{t})=\frac{R_{o}}{V_{p} K_{p}}+\frac{K_{b} X_{p o}-K_{b}\left(X_{p o}+X_{b o}\right)+R_{o}}{V_{p}\left(K_{b}-K_{p}\right)} e^{-K_{b} t}+\frac{K_{p}^{2} X_{p o}-K_{b} K_{p}\left(X_{p o}+X_{b o}\right)+R_{o} K_{b}}{V_{p} K_{p}\left(K_{p}-K_{b}\right)} e^{-K_{p} t}
$$

$$
\begin{aligned}
& \text { Incorporation } R_{o}=d C_{a}, K_{b}=\frac{d}{V_{b}} \text { and } K_{\mathrm{p}}=\frac{d}{V_{P}} \text { in the equation A4 gives } \\
& C_{p}(t)=C_{a}+\left(C_{b o}-C_{a}\right) \frac{V_{b}}{V_{b}-V_{p}} e^{-\frac{d}{V_{b}} t}+\left[\left(C_{p o}-C_{a}\right)-\left(C_{b o}-C_{a}\right) \frac{V_{b}}{V_{b}-V_{p}}\right] e^{-\frac{d}{V_{p}} t}
\end{aligned}
$$

When $\mathrm{t}=0, \mathrm{C}_{\mathrm{p}}(\mathrm{t})=\mathrm{C}_{\mathrm{po}}$. When $t \rightarrow \infty, \mathrm{C}_{\mathrm{p}}(\mathrm{t})=\mathrm{C}_{\mathrm{a}}$

The following differential equations may be written for the change in amount of drug in the central and two peripheral compartments with time:

$$
\begin{aligned}
& \frac{d X_{1}}{d t}=K_{p} X_{p}+K_{21} X_{2}+K_{31} X_{3}-\left(K_{12}+K_{13}+K_{10}\right) X_{1} \\
& \frac{d X_{2}}{d t}=K_{12} X_{1}-K_{21} X_{2} \\
& \frac{d X_{3}}{d t}=K_{13} X_{1}-K_{31} X_{3}
\end{aligned}
$$

The Laplace transform of equations A6 and A7 are

$$
\overline{X_{2}}=\frac{K_{12} \overline{X_{1}}}{S+K_{21}}
$$


$\overline{X_{3}}=\frac{K_{13} \overline{X_{1}}}{S+K_{31}}$

Incorporation $\overline{X_{2}}, \overline{X_{3}}$ and $\overline{X p}$ in the equation A5 gives

$$
\begin{gathered}
\overline{X_{1}}\left[S+\left(K_{12}+K_{13}+K_{10}\right)-\frac{K_{21} K_{12}}{S+K_{21}}-\frac{K_{31} K_{13}}{S+K_{31}}\right]=\frac{d}{V_{p}} \frac{X_{p o}\left[S^{2}+K_{b}\left(1+\frac{X_{b o}}{X_{p o}}\right) S+\frac{R_{o} K_{b}}{X_{p o}}\right]}{S\left(S+K_{b}\right)\left(S+K_{p}\right)} \\
\text { Let } \square= \\
=\frac{S+\left(K_{12}+K_{13}+K_{10}\right)-\frac{K_{21} K_{12}}{S+K_{21}}-\frac{K_{31} K_{13}}{S+K_{31}}}{\left(S+K_{21}\right)\left(S+K_{31}\right)}
\end{gathered}
$$

Since

$$
\begin{aligned}
& \alpha+\beta+\pi=K_{10}+K_{12}+K_{13}+K_{21}+K_{31} \\
& \alpha \beta+\alpha \pi+\beta \pi=K_{10} K_{21}+K_{13} K_{21}+K_{10} K_{31}+K_{21} K_{31}+K_{31} K_{12} \\
& \alpha \beta \pi=K_{21} K_{31} K_{10}
\end{aligned}
$$

Therefore $\square=\frac{(S+\alpha)(S+\beta)(S+\pi)}{\left(S+K_{21}\right)\left(S+K_{31}\right)}$

Rearranging equation A10 gives

$$
\overline{X_{1}} \frac{(S+\alpha)(S+\beta)(S+\pi)}{\left(S+K_{21}\right)\left(S+K_{31}\right)}=\frac{d}{V_{p}} X_{p o}\left(\frac{(S+V)(S+W)}{S\left(S+K_{b}\right)\left(S+K_{p}\right)}\right)
$$

Rearranging equation A15 gives

$$
\begin{aligned}
& \overline{X_{1}}=\frac{\frac{d X_{p o}}{V_{p}} \frac{(S+V)(S+W)}{S\left(S+K_{b}\right)\left(S+K_{p}\right)}}{\frac{(S+\alpha)(S+\beta)(S+\pi)}{\left(S+K_{21}\right)\left(S+K_{31}\right)}} \\
& K_{b}=K_{21}, K_{p}=K_{31},(S+V)(S+W)=(S+\alpha)(S+\beta) \text {, then: } \\
& \overline{X_{1}}=\frac{d X_{p o}}{V_{p} S(S+\pi)}=\frac{d C_{p o}}{S(S+\pi)}
\end{aligned}
$$

Solving for $\mathrm{X}_{1}(\mathrm{t})$ employing a table of Laplace transforms, gives 


$$
X_{1}(t)=\frac{d C_{p o}}{\pi}\left(1-e^{-\pi t}\right)
$$

$C_{p o}=\frac{C_{a} \pi}{K_{10}} \quad$ in the equation A18 gives:
Incorporation

When $t \rightarrow \infty, C_{1}(\infty)=\frac{C_{a} d}{K_{10} V_{1}}=C_{\mathrm{ss}}$, therefore

Rearranging equation 3 gives

$$
C_{a}=\frac{C_{s s} V_{1} K_{10}}{d}
$$

Let $K_{p}=K_{21}$ and $K_{b}=K_{31}$, then:

$$
V b=\frac{d}{K_{31}}
$$

$$
V p=\frac{d}{K_{21}}
$$

Since

$$
\begin{aligned}
& S^{2}+(V+W) S+V W=S^{2}+(\alpha+\beta) S+\alpha \beta \\
& S^{2}+\frac{K_{b}\left(X_{p o}+X_{b o}\right)}{X_{p o}} S+\frac{K_{b} R_{o}}{X_{p o}}=S^{2}+(\alpha+\beta) S+\alpha \beta
\end{aligned}
$$

Rearranging equation A19 and A20 gives

$$
\begin{aligned}
& \alpha+\beta=\frac{K_{b}\left(X_{p o}+X_{b o}\right)}{X_{p o}} \\
& \alpha \beta=\frac{K_{b} R_{o}}{X_{p o}}
\end{aligned}
$$

Rearranging equation A22 gives

$$
X_{p o}=\frac{K_{b} R_{o}}{\alpha \beta}=\frac{K_{31} C_{a} d}{\frac{K_{21} K_{31} K_{10}}{\pi}}=\frac{C_{a} d \pi}{K_{21} K_{10}}=\frac{C_{a} d K_{31}}{\alpha \beta}
$$


Since $\alpha \beta \pi=K_{21} K_{31} K_{10}$ :

$$
C_{p o}=\frac{X_{p o}}{V_{p}}=\frac{C_{a} d \pi}{V_{p} K_{21} K_{10}}=\frac{C_{a} d \pi}{\frac{d}{K_{21}} K_{21} K_{10}}=\frac{C_{a} \pi}{K_{10}}
$$

(Equation 8)

Rearrange equation A21 gives

$$
X_{b o}=\frac{X_{p o}\left(\alpha+\beta-K_{b}\right)}{K_{b}}
$$

which can be written in concentration terms:

$$
C_{b o}=\frac{X_{p o}}{V_{b}}\left(\frac{\alpha+\beta}{K_{b}}-1\right)=\frac{C_{a} d K_{31}}{\alpha \beta}\left(\alpha+\beta-K_{31}\right)
$$

It is evident that:

$\mathrm{X}_{\mathrm{a} 0}=\mathrm{C}_{\mathrm{a}} \times \mathrm{V}_{\mathrm{a}}$

$\mathrm{X}_{\mathrm{b} 0}=\mathrm{C}_{\mathrm{b} 0} \times \mathrm{V}_{\mathrm{b}}$

(Equation 7)

$\mathrm{X}_{\mathrm{p} 0}=\mathrm{C}_{\mathrm{p} 0} \times \mathrm{V}_{\mathrm{p}}$

(Equation 10)

Thus, the proof of equations (1) - (10) in Table I is completed. 